\title{
Optimising Production through Intelligent Manufacturing
}

\author{
Isaac O. Olalere ${ }^{1, *}$, and Oludolapo A. Olanrewaju ${ }^{2}$ \\ ${ }^{1}$ Durban Univ. of Technology, Industrial Engineering Department, 4001, Durban, South Africa \\ ${ }^{2}$ Durban Univ. of Technology, Industrial Engineering Department, 4001, Durban, South Africa
}

\begin{abstract}
Intelligent manufacturing system (IMS) has been the focus of most industries since Industry 4.0 revolution. IMS is being implemented through the integration of Internet of Things, (IoT), Cyber-Physical Systems (CPS), digital twin and big data analytics to optimize production through smart manufacturing. This research presents a conceptual approach of an adaptive clustering algorithm (ACA) for advanced manufacturing decision-making for smart machining manufacturing. The work considers product monitoring and assessment, machine health and operating parameters monitoring, as an important factor for intelligent decision making on a machining production line through the developed cyber twin of the machine tool for production optimisation. Cyber twin of the machine tool is developed which runs on a realtime sequence with the physical asset fussed with smart sensors and controllers enabled with cloud computing, IoT and data analytics. The ACA enables resources monitoring, production monitoring, machine condition monitoring, cloud feedback notification, product monitoring, and assessment, for intelligent decision-making from a cluster of similar machines using ANN clustering tool for self-aware, self-predict and self-reconfiguration in a smart machining production line to detect a cutting tool chipping of less than $0.25 \mathrm{~mm}$ size. The method is proposed to optimise production by increasing productivity through intelligent decision and prediction for tool change, tool failure, maintenance, adjustment of operating parameters.
\end{abstract}

\section{Introduction}

Intelligent manufacturing systems is targeted at transforming and upgrading manufacturing technologies by Cyber-physical systems (CPS), the internet of Things (IoT), and cloud computing[1]. In recent technologies, systems have been equipped with the ability of monitoring the physical processes and make smart decisions through co-operation with humans, machines, and intelligent devices through real time communication[2]. A new generation of machine tool i.e. machine tool 4.0 known as cyber physical machine tool (CPMT) has been proposed, which is intended to integrate machine tool, machining processes, computation and networking for monitoring and control of machining processes with feedback loops [3]. Different implementation of sensors, data acquisition systems, computer network and cloud computing have prepared infrastructure for designing and implementing intelligent manufacturing systems. The implementation of this technology has therefore resulted into generation of huge amount of data from these intelligent devices. Hence, big data is the information asset characterized by such a high volume, velocity and variety to require specific technology and analytical methods for its transformation into value[4]. Advances in technologies have introduced three main advanced manufacturing technologies : intelligent manufacturing, IoT-enabled manufacturing and cloud manufacturing. In 2012, GE developed the idea of the Industrial Internet of Things
(IIoT), suggesting that intelligent machines, advanced analytics, and connected people are the key elements of future manufacturing in order to enable smarter decisionmaking by humans and machines. Yuqing [5] determined that the surface roughness or finish of a workpiece is highly dependent on tool wear which also increases the cutting force during milling operation. This implies that tool wear increases the surface roughness and corresponding increase in cutting force.

Intelligent manufacturing requires certain underpinning technologies in order to enable devices or machines to vary their behaviours in response to different situations and requirements based on past experiences and learning capacities[6]. An intelligent manufacturing system that adopt motorized high speed spindle on machine tools based on reliability engineering was propose by Hsun-Fu et al. [7].

This study aimed at conceptualizing a smart-decision making algorithm of an intelligent manufacturing system through integration of recent technologies, such as CPS, IoT, cloud computing, digital twin and real-time communication and information learning for intelligent production system. An intelligent collection and monitoring system of processing information of a CNC machine tool was developed for real-time performance analysis, machining efficiency and fault statistics. The next section reviews the recent technologies being gradually adopted for intelligent manufacturing. 


\section{Cyber-Physical Systems}

CPS is a mechanism through which physical objects and software are closely intertwined, enabling different components to interact with each other in a myriad of ways to exchange information [8]. A CPS-enabled system consists of networked smart interactions that are designed and developed with physical input and output, together with their intertwined cyber services such as control algorithm, decision making algorithm, and computational capacities. The CPS framework unlike Physical Health Monitoring (PHM) systems that is developed from data collected through experiments, performs a self-maintenance and self-aware capabilities for intelligent decision-making. Guanghui [9] proposed a general frame work for knowledge drive digital twin manufacturing cell (ICDTMC) which supports intelligent manufacturing through intelligent perceiving, simulating, understanding, predicting, optimizing and controlling strategy. Therefore, it is important to consider the number of sensors incorporated into the CPS.

\section{Internet of Things}

IoT is the technology that enables global network of machines and devices capable of interacting with each other. IoT-enabled manufacturing features real-time data collection and sharing among various manufacturing resources such as machines, workers, materials, and jobs [10]. IoT capabilities enable devices to interact, communicate, evaluate, and capture real-time data, and sends decision to the actuators. All IoT devices adopts a mechanism to send or receive data such as wired or wireless, Bluetooth, cellular network and many more[11]. Communication between machines and smart devices such as sensors, controllers are done via the cloud services. This IoT-enabled controller is programmed to capture signals from the machines or inter-connected systems through network of sensors, signal processing and decision making. Several real-life cases of IoTenabled manufacturing have been developed. An Elevator system was being monitored by[12], for early detection of fault remotely through IoT enabled devices, that captures the data using cloud services and the machine condition together with the maintenance decision communicated to the operators and maintenance team.

\section{Cloud Computing}

Cloud computing technology is a powerful and vast computing capabilities with greater deal of computational infrastructure that helps to overcome some limitations of IoT. Cloud computing has given rise to higher scale of being generated. The scale of data being generated through cloud computing has led to the concept of big data.

Some of the advantages of cloud computing includes the ability to visualize resources, execute parallel processing, scrutiny and data service integration with scalable data storage[13]. IoT is limited by storage and processing capacity, while cloud computing on the other hand has unlimited capacities both for storage and process capacity, hence, a novel paradigm that integrates cloud and IoT emerged called Cloud-IoT [14]. Integrating cloudIot into manufacturing would create a high level of manufacturing intelligence that would enhance productivity, reduce machine downtime through effective decision making via machine learning and data analytics.

A new generation of tool condition monitoring (TCM) that detects tool wear of approximately $0.25 \mathrm{~mm}$ accurately and also predict sudden tool failure using advanced signal processing techniques to multi-sensor feedback signals was developed by Hassan [15]. Maojun[16] investigated the surface integrity of carbon fibre reinforced plastics (CFRP) composite and tool wear at variable feed rate while decreasing the drill feed from maximum of $0.3 \mathrm{~mm} / \mathrm{rev}$ to $0.01 \mathrm{~mm} / \mathrm{rev}$ for the final $2 \mathrm{~mm}$ of hole dept, so as to reduce thrust force generated by worn drills that in turn reduces the hole surface quality. Hocheng et al.[17] applied laser-light scattering to monitor the tool wear using an absorber or detector to measure the intensity of the scattered light beams as different incident angles and noted that the energy intensity collected by detector increases with tool wear

\section{Methodology}

The research method develops a cyber physical machine tool architecture that integrates real-time machining data (twin), laser-light scattering method for surface finish of workpiece and intelligent algorithm to machine tool cyber twin for cutting tool wear/chipping capturing.

- Machine tool Digital Twin development using ANSYL software, sensors, IoT enabled controllers

- Data learning and training for detection of tool wear/chipping of less than $0.25 \mathrm{~mm}^{2}$.

The first step is developing the experimental set-up and design of experiments. The experiment is aimed at monitoring and determining the cutting tool condition through intelligent feedback loops from decision algorithms incorporated to the tool cyber twin based on both the machine operation parameters (machining operation), tool condition and the workpiece. Research to determine cutting tool condition have been done but however, adopts either workpiece assessment or the machine tool operating conditions in assessing the tool condition. This research aimed at integrating both the workpiece assessment, machine tool operating condition and the real-time tool condition assessment implemented through the machine tool cyber twin into artificial intelligent decision algorithm for the feedback loop for self-adaptivity and self-reconfiguration. Two independent machine tool clusters may be integrated for adaptive learning and self-adjustment from clusters of similar machines which has been proposed but never been done. The first step involves identifying the machine tool, cutting tool, workpiece material, sensor selection and assessment of the cutting tool condition. 
This also includes identifying the machining operating parameters to be incorporated into the machine tool cyber twin and the workpiece characteristics for realtime implementation of tool condition monitoring system for smart manufacturing

The second step involves a careful selection of the sensors for tool and workpiece condition monitoring and installing the sensors network on the machine tool. Machine tool operating parameters such as spindle axial vibrations, acoustics emission and drive motor power to determine the cutting forces, laser-light beam and absorber for workpiece surface finish, infra-red camera for tool image capturing, spindle speed sensor. Wireless sensors are used to facilitate the network connections to the processor or controller that support the smart manufacturing system. The smart devices are IoT enabled in order to accommodate for cyber systems and cloud computing and fusion with the digit twin.

The third step is the development of the machine tool digital twin that simulates the physical behavior of the machine tool during operation. This requires the configuration of the sensors and smart devices to a cyber digital machine twin developed using a software such as ANSYL, enabled through IoT connectivity. The machine tool digital twin is developed to imitate and mimic the real physical machine tool.

The fourth step is tool breakage/chipping characterization and detection during intermittent machining process. This step involves experimental investigation of monitored signals and its ability to indicate tool chipping or breakage. This involves analysis and signal processing of monitored parameters for tool condition monitoring. Overall experiment must also detect a small chipping of less than $0.25 \mathrm{~mm}$ size of the cutting tool. Artificial signals might be used at this stage to also test the approach for feasibility.

The fifth step is the development of a real-time tool chipping monitoring system. This involve several methods for feature extraction and analysis to garner relevant and important information concerning the tool condition. Signal processing approach is also discussed, and analysis of data is also considered at this stage for intelligent decision making for the feedback loop to the cyber twin for self-reconfiguration. Different classification methods and pattern recognition of the extracted features are considered at this level selflearning and self-adaptivity.

The sixth step involves the experimental testing and validation of the online implementation of the Cyber Twin with tool chipping detection system. This involves analysis of the results, learning algorithm and its accuracy.

The method also considers two clusters of similar machine tool for adaptive learning and selfreconfiguration.

\subsection{Adaptive Clustering for Self-Aware Machine and Product Analysis}

The efficiency and effectiveness of the proposed IMS structure lies on the performance of the data analysis functions deployed in the cyber level. This level interprets what the condition of the manufacturing system is as well as well as determine self-adjustment decision regarding the machine health condition as well as the process appraisal through product quality monitoring. Some sensors are installed to monitor the manufacturing process as well as the quality output of the product being produced. [18] proposed a service encapsulated virtual model that combines basic attributes of a physical object, the quality of service of the physical object, its capacity function and the status.

This approach however integrates product quality learning into the adaptive clustering algorithm for accessing the machine health condition, monitoring the manufacturing process, service distribution and sharing for advanced manufacturing decision-making.

The first level is the data acquisition that deals with extracting possible signals from the manufacturing systems including machine parts, accessories using smart sensors that are IoT capability. The next level is the datato- information level which is carried out by the IoT enabled controller. The signals are converted to information for smart analysis for process monitoring, component health, degradation and performance prediction, data correlation and product quality monitoring.

The cyber level is required to autonomously accumulate system knowledge and learn based on data collected from a group machines, products, similar production lines. Machine condition, material handling condition, degradation pattern, failure modes, product quality threshold, are some system knowledge which is used for re-configuration functions for optimization and intelligent manufacturing decision making. Unsupervised learning algorithms can be used to create clusters of for different working regime. Self-Organizing Map (SOM) and Gaussian Mixture Model (GMM) are both good algorithms for unsupervised learning which could be adopted.

An Adaptive clustering algorithm methodology is proposed, that makes use of on-line update mechanism for comparing the latest input parameter against the existing cluster, carrying out self-identification of the most similar cluster to the input using multidimensional distance measurement. The algorithm presents a cluster matching approach that identifies a cluster matching with the new input and update latest information from the new sample.

The next scenario may be presented when there is no similar existing cluster with the new input. In this case, the sample is held on stand-by for a while till a level of similar samples to this new input is recorded, a new cluster is then created as a new machine behavior.

\subsection{Turning Operation Case Study}

This section conceptualized the application of adaptive algorithm on flexible CNC machining manufacturing systems, for production of GT parts. FMS is capable of producing variety of parts, through computer control of the NC machines. It is a highly automated GT machine 
cell, comprising of several workstations that carry out processes which are interconnected by automated material handling and storage system that are controlled by a distributed computer system. Adaptive clustering algorithm applies cloud computing, IoT, Big Data analytics, and artificial intelligence to monitor, capture, learn and classify several machine specific patterns, health values, working regimes, material handling and storage system as well as the output/product attributes in order to develop a knowledge base for smart decision making from clusters of machines, components, workcarriers, conditions, and product characteristics.

\section{Conclusion}

Intelligent manufacturing system is generally finding its bare in most modern industry 4.0 industries. The combined technology and concept of IoT, Cloud Computing, data analytics and cyber-physic systems formed the underlying technology for the proposed adaptive clustering algorithm for IMS. The adaptive clustering algorithm can be applied to a machining production line for self-adjusting, self-aware, selfconfigure, and self-predict attributes for intelligent manufacturing. The application of the algorithm would reduce the frequency of downtime of the production line, detect the cutting tool condition, increase the quality of the product output, and also increase the system's reliability. An optimized model, considering the machine health, process parameters, and product assessment, can therefore be derived from the application of the adaptive clustering algorithm on a fully automated production line in an Intelligent Manufacturing System, IMS.

\section{References}

1. Lee, J., B. Bagheri, and H.-A. Kao, A cyberphysical systems architecture for industry 4.0based manufacturing systems. Manufacturing Letters, 2015. 3: p. 18-23.

2. Zhong, R.Y., et al., Intelligent manufacturing in the context of industry 4.0: a review. Engineering, 2017. 3(5): p. 616-630.

3. Cai, Y., et al., Sensor data and information fusion to construct digital-twins virtual machine tools for cyber-physical manufacturing. Procedia Manufacturing, 2017. 10: p. 1031-1042.

4. De Mauro, A., M. Greco, and M. Grimaldi, A formal definition of Big Data based on its essential features. Library Review, 2016. 65(3): p. 122-135.

5. Zhou, Y. and W. Xue, Review of tool condition monitoring methods in milling processes. The
International Journal of Advanced Manufacturing Technology, 2018. 96(5-8): p. 2509-2523.

6. Miorandi, D., et al., Internet of things: Vision, applications and research challenges. Ad hoc networks, 2012. 10(7): p. 1497-1516.

7. Chiang, H.-F., et al. Study of Motorized Spindle Reliability Monitoring. in 2018 International Conference on Applied Electronics (AE). 2018. IEEE.

8. Khaitan, S.K. and J.D. McCalley, Design techniques and applications of cyberphysical systems: A survey. IEEE Systems Journal, 2015. 9(2): p. 350-365.

9. Zhou, G., et al., Knowledge-driven digital twin manufacturing cell towards intelligent manufacturing. International Journal of Production Research, 2019: p. 1-18.

10. Zhong, R.Y., X. Xu, and L. Wang, IoT-enabled smart factory visibility and traceability using laserscanners. Procedia Manufacturing, 2017. 10: p. 114.

11. Olalere, I.O., M. Dewa, and B. Nleya. Remote Condition Monitoring of Elevator's Vibration and Acoustics Parameters for Optimised Maintenance Using IoT Technology. in 2018 IEEE Canadian Conference on Electrical \& Computer Engineering (CCECE). 2018. IEEE.

12. Olalere, I.O. and M. Dewa, Early fault detection of elevators using remote condition monitoring through IoT technology. South African Journal of Industrial Engineering, 2018. 29(4): p. 17-32.

13. Hashem, I.A.T., et al., The rise of "big data" on cloud computing: Review and open research issues. Information systems, 2015. 47: p. 98-115.

14. Botta, A., et al., Integration of cloud computing and internet of things: a survey. Future generation computer systems, 2016. 56: p. 684-700.

15. Hassan, M., et al., Intelligent machining: real-time tool condition monitoring and intelligent adaptive control systems. Journal of Machine Engineering, 2018. 18.

16. Li, M., et al., Study on tool wear and workpiece surface integrity following drilling of CFRP laminates with variable feed rate strategy. Procedia CIRP, 2018. 71: p. 407-412.

17. Hocheng, H., et al., Tool wear monitoring in singlepoint diamond turning using laser scattering from machined workpiece. Journal of Manufacturing Processes, 2018. 31: p. 405-415.

18. [18] Qi, Q., et al., Digital Twin Service Towards Smart Manufacturing. Procedia CIRP, 2018. 72(1): p. 237-242. 